\title{
Incidencia del mindfulness en el estrés académico en estudiantes universitarios: Un estudio controlado
}

\author{
Incidence of mindfulness in academic stress in university students: \\ a controlled study
}

\author{
Luis Armando Oblitas Guadalupe \\ Duber Enrique Soto Vásquez, \\ José Carlos Anicama Gómez \\ Adolfo Alfredo Arana Sánchez \\ Universidad Autónoma del Perú
}

Rec (8 de noviembre de 2018) Acept (13 de junio de 2019)

\begin{abstract}
Resumen
El mindfulness es un método cognitivo que consiste en prestar atención plena al cuerpo, respiración, pensamientos, emociones, y conductas, que se utiliza para reducir el estrés, dolor, ansiedad y depresión, el cual cuenta con algunas evidencias clínicas. Recientemente se está evaluando sus aplicaciones en el ámbito universitario, con resultados aún no concluyentes. El propósito del estudio fue evaluar la incidencia de un programa de ocho semanas del mindfulness en la reducción del estrés académico en una muestra de 54 estudiantes universitarios, con grupo de control aleatorio, y con medidas de pre-posprueba utilizando el Inventario CISCO de Estrés Académico. Se encontró que no existe diferencias estadísticamente significativas en los niveles de estrés del grupo que recibió el programa de mindfulness comparado con los que no recibieron el entrenamiento. El resultado es congruente con algunos hallazgos que no atribuyen eficacia de la atención plena en el manejo del estrés académico.
\end{abstract}

Palabras clave: mindfulness, estrés académico, universitarios

\begin{abstract}
Mindfulness is a cognitive method that consists of giving full attention to the body, breathing, thoughts, emotions, and behaviors, which is used to reduce stress, pain, anxiety and depression, which has some clinical evidence. Recently, its applications in the university field are being evaluated, with results that are not yet conclusive. The purpose of the study is to evaluate the incidence of an eight-week program of mindfulness in reducing academic stress in a group of 54 university students, with a randomized control group, and with pre-post-test measures using the CISCO Academic Stress Inventory. It was found that there are no statistically significant differences in the stress levels of the group that received the der mindfulnes program compared to those who did not receive the training. The result is congruent with some findings that do not attribute efficiency of mindfulness in the management of academic stress.
\end{abstract}

Keywords: mindfulness, academic stress, university.

Correspondencia: Dirección de Investigación, Universidad Autónoma del Perú. Km. 16.3 Carretera Panamericana Sur, Villa El Salvador, Lima, Perú. E-mail: luis.oblitas@autonoma.pe 


\section{Introducción}

El mindfulness (MF) o atención plena (AP) a menudo se refiere a prácticas específicas utilizadas para enfocar la atención de una persona (meditación, yoga, respiración, concentración de un solo punto, en un objeto, etcétera) y se caracteriza por la intencionalidad y la observación sin prejuicios de la experiencia. La AP se asocia con la espiritualidad oriental, pero las aplicaciones puramente seculares de mindfulness se han explorado cada vez más en entornos tan variados como en la clínica, el lugar de trabajo, las instituciones correccionales y las instituciones educativas (Broderick \& Jennings, 2012)

El MF es una estrategia cognitiva atencional que en la actualidad se utiliza para entrenar la regulación de la atención con el propósito de reducir el estrés y promover la salud psicológica, y recientemente se ha convertido en una técnica muy popular en las universidades para reducir el estrés producido por la vida escolar, las tareas y los exámenes (Galante et al., 2018). La aceptación del enfoque podría explicarse en gran parte por la percepción del entrenamiento de la atención plena como una habilidad más que como una intervención de salud mental, de fácil aprendizaje y aplicación, que contribuye al bienestar psicológico en los estudiantes (Barnes, Hattan, Black \& Schuman, 2017).

\section{Evidencias que apoyan la efectividad del mindfulness}

En un estudio de meta-análisis realizada por Regehr, Glancy \& Pitts (2013), se eligieron 24 estudios, que incluyeron 1431 estudiantes universitarios, y encontraron que los programas cognitivos, conductuales y de mindfulness se asociaron con niveles más bajos de ansiedad en estudiantes universitarios. Schonert \& Lawlor (2010), aplicaron un programa de MF a estudiantes $(n=246)$, tres veces al día durante seis sesiones y obtuvieron mejoras significativas en optimismo, conductas pro-sociales, auto-concepto y afecto positivo. Ramasubramania (2017), evaluaron el impacto de curso de comunicación basado en MF en estudiantes universitarios, y obtuvieron un incremento de emociones positivas y reducción del estrés percibido en el grupo de mindfulness $(n=24)$, en comparación con el grupo control $(\mathrm{n}=20)$.

Dvořáková, et al. (2017), evaluaron el MF para promover salud y bienestar en 109 estudiantes universitarios, encontrando un aumento significativo en la satisfacción con la vida y una disminución también relevante de la depresión y la ansiedad. Di Pierdomenico, Kadziolka \& Carlin (2017), examinaron en 55 estudiantes universitarios las relaciones entre MF, afrontamiento y reactividad, obteniendo una correlación negativa entre el uso de sustancias y el entrenamiento en MF. Pidgeon \& Pickett (2017), encontraron en 122 estudiantes universitarios australianos que los puntajes de mayor resiliencia se asocian con el MF, con mejores puntajes de afrontamiento adaptativo, y un nivel reducido de ansiedad.

Miller, Borsatto \& Al-Salom (2018), compararon los efectos de recibir o no una breve práctica diaria de cinco minutos basada en MF en 88 estudiantes canadienses; al final del semestre, el grupo de mindfulness informó haber experimentado mejoras en la emocionalidad positiva y menos episodios de distracción cognitiva que los del grupo de control. Martin (2018), encontró que el MF repercute en una mayor disponibilidad y acercamiento con los compañeros de aula, así como en la generación de experiencias de auto-regulación emocional en estudiantes comunitarios. Reavley (2018), asignó al azar a 616 estudiantes para recibir el curso de MF $(n=309)$ y a un grupo en lista de espera $(n=307)$, encontrando que el mindfulness redujo el estrés durante el período de examen en comparación con el grupo en lista de espera

Parcover, Coiro, Finglass \& Barr (2017) demostraron que el MF produjo una reducción en el estrés, ansiedad, depresión en 78 estudiantes universitarios en comparación con un grupo control $(n=109)$. Greeson, Juberg, Maytan, James \& Rogers (2014), realizaron un estudio aleatorio controlado utilizando un programa de MF con 90 estudiantes universitarios, encontrando una disminución significativa del estrés, reducción de los problemas para dormir, mejoras en la atención plena y la auto-compasión. Oman, Shauna \& Shapiro (2010), evaluaron el impacto del MF en 30 estudiantes universitarios, asignados al azar a dos condiciones, y obtuvieron diferencias significativas en la reducción del estrés, perdón y esperanza.

Caldwell, Harrison, Adams, Quin \& Greson (2010), reportaron el impacto del MF en 166 estudiantes universitarios durante 15 clases semanales en el incremento de la atención y la percepción del estrés. Li, Kee \& Lam (2018), evaluaron el impacto de una breve inducción de mindfulness para mejorar el sueño de los atletas antes del sueño y después del entrenamiento nocturno $(\mathrm{n} 1=32)$, en comparación con el grupo control $(\mathrm{n} 2=31)$, 
encontrando que el MF redujo la excitación previa al sueño y mejoró el nivel de reposo y la calidad general del sueño. Galante et al. (2018), en un estudio aleatorio con 616 estudiantes universitarios (experimental, $\mathrm{n}=309$; control, $n=307$ ), administraron un programa de mindfulness de 8 semanas encontrando reducción el distrés emocional durante el periodo de exámenes.

Muñoz, Hoppes, Hellman, Brunk, Bragg \& Cummins (2016), utilizaron un diseño cuasi experimental aleatorio con grupo de comparación ( $\mathrm{n}=46 ; 23$ grupo de meditación y 23 grupo control), indicando que el MF produjo un incremento significativo de la esperanza mediante la reducción del estrés. Tarrasch, Berman \& Friedman (2016), encontraron que MF mejora la lectura, la atención y el bienestar psicológico en 19 adultos con dislexia y con déficit de atención. Shaidi, Akbari \& Zargar (2017), lograron que el MF influya significativamente sobre la regulación emocional y la ansiedad ante los exámenes en 50 estudiantes asignados al azar a dos condiciones.

Ingram, Breen \& van Rhijn (2017) en un estudio exploratorio con 15 estudiantes universitarios, demostraron que el MF fomenta la auto-reflexión, auto-conocimiento, relajación, y facilita la discusión y el aprendizaje. Gouda, Luong, Schmidt \& Bauer (2016), en un estudio con 29 estudiantes universitarios ( $n=15$, grupo experimental; $n=14$, grupo control), encontraron que el MF produce mejoras significativas en la reducción del estrés, la autorregulación, la autoeficacia y en los problemas interpersonales. Ching, Koo, Tsai, \& Chen 2015), evaluaron los efectos de un curso semestral en 152 estudiantes universitarios taiwaneses y se le comparó con una muestra de control de 130, obteniendo que el MF fue capaz de mejorar la efectividad del aprendizaje y los aspectos de atención y memoria del desempeño cognitivo.

Ahmad, Sharif, Akbar, \& Alavi (2014), investigaron la incidencia del MF en 273 estudiantes universitarios, logrando aumentar la condición de salud, mejoró la calidad de la salud mental, e incrementó la motivación por los estudios. Verweij, van Ravesteijn, van Hooff, Lagro, \& Speckens (2018), aplicaron un programa de MF para los residentes de medicina, quienes indicaron que aumentaron su conciencia y autorreflexión en el trabajo, y fueron más receptivos hacia sí mismos y hacia sus limitaciones, más resilientes, mejoraron el autocuidado y el equilibrio entre el trabajo y la vida. Ghaeni, Hashemian, Mojtabaei, Majdara, \& Aghabeiki (2017), incrementaron en forma significativa con el MF la asertividad y ansiedad en 45 mujeres universitarias islamistas asignadas al azar a grupos experimental, control y placebo.

Ali, Weiss, Dutton, McKee, Jones, Kashikar, Silverman \& Shapiro (2017), lograron con el MF un impacto positivo en las actividades de la vida diaria, dolor y ansiedad en 18 adolescentes con dolor crónico generalizado. D’Ábundo, Cara \& Kelly (2016), realizaron un estudio con 225 estudiantes universitarios con un programa de MF logrando una reducción significativa del estrés y mejora en el bienestar psicológico. Cho, Ryu, Noh \& Lee (2016), evaluaron una condición de respiración consciente, una condición de reevaluación cognitiva, y grupo de control. Los resultados mostraron que tanto la práctica de respiración consciente como la práctica de revalorización cognitiva contribuyeron a la reducción la ansiedad.

\section{Evidencias que cuestionan la efectividad del mindfulness}

Los estudios han demostrado la eficacia del entrenamiento en MF en la disminución de los síntomas de los trastornos psicológicos comunes, como el estrés, la ansiedad y la depresión (Goyal, Singh, \& Sibinga, 2014); sin embargo, existen aún pocas pruebas definitivas de la efectividad en la prevención de trastornos psicológicos comunes en estudiantes universitarios, y ningún estudio ha monitoreado hasta el momento activamente los posibles efectos adversos.

Los estudios previos se basaron principalmente en estudiantes de servicios de salud, estudios que en su gran mayoría adolecían de poder estadístico, grupos de control, protocolo establecido, y hallazgos claros. Además, existen preocupaciones sobre las mediciones múltiples, el sesgo de lealtad de los investigadores con los profesores que actúan como investigadores, el análisis y el tratamiento inadecuados de los datos, y los tiempos de seguimiento poco realistas (Lo, Waterland, \& Todd, 2018; O’Driscoll, Byrne, McGillicuddy, Lambert, \& Sahm, 2017; McConville, McAleer, Hahne, 2017).

Lynch, Gander, Nahar, Kohls, \& Walach (2018), en un estudio aleatorio y controlado con grupo control $(\mathrm{n}=10)$ utilizaron un programa de MF de 8 semanas con estudiantes universitarios y encontraron una disminución significativa de ansiedad, depresión, estrés percibido al comparar los datos del grupo experimental $(\mathrm{n}=14)$, pero los resultados no podrían ser generalizados ya que la muestra fue pequeña. Kreplin, Farías \& Brazil (2018), revisaron la literatura reciente y reportaron un efecto moderado del MF en la pro-socialidad en adultos sanos, 
aunque los cambios no fueron significativos. Golberg, Tucker, Greene, Simpson, Kearney, \& Davidson (2017), realizaron una revisión de la literatura (2000-2016), y seleccionados 142 estudios clínicos aleatorizados de intervenciones basadas en MF para muestras con un trastorno clínico. No obtuvieron evidencias de mejoras en ningún indicador de metodología y calidad de los estudios.

De Vibe, Solhaug, Tyssen, Friborg, Rosenvinge, \& Sørlie (2013), encontranron efectos moderados posteriores a la intervención del MF sobre la ansiedad psicológica, un pequeño efecto sobre el bienestar psicológico, y sin mayor incidencia sobre la atención plena $(n=120)$. Hj, Alavi, Mehrinezhad, \& Ahmadi (2018), con un grupo de 384 estudiantes de pregrado en Malasia encontraron un resultado dispar: con el análisis correlacional obtuvieron una relación significativa entre estrés académico, la autorregulación y MF; y, utilizando el análisis de mediación del SPSS, el MF no demostró el rol de mediador en la investigación. Zenner, Herrnleben, \& Walach (2014), realizaron una revisión de la literatura para evaluar los efectos del MF en las escuelas; encontraron hallazgos prometedores en niños y jóvenes, en la mejora del rendimiento cognitivo y la resiliencia ante el estrés, sin embargo, la diversidad de las muestras de estudio, la variedad en la implementación, y una amplia gama de instrumentos utilizados, hacían imposible evaluar los datos.

Holland, Dooley, Fedock, Ferebee \& Bailey (2017), encontraron que no existía relación entre edad, género y etnia con el mejoramiento del pensamiento crítico mediante la práctica del MF en 22 estudiantes norteamericanos. Semple, Droutma \& Reid (2017), evaluaron reflexivamente varios programas de mindfulness en las escuelas sobre algunas cuestiones metodológicas aprendidas hasta el momento sobre la investigación y de las experiencias del mundo real, señalando que a pesar de estar bien implementados los programas de MF estos tienen poco o ningún apoyo de investigación para evaluar su efectividad. Doss \& Bloom (2018), implementaron un programa de MF de 30 días a estudiantes sobre dotados para aprender técnicas para aliviar el estrés, las tendencias perfeccionistas y la ansiedad. Algunos alumnos fueron capaces de afrontar los problemas, mientras que otros les costó comprender los objetivos del programa.

Galante et al. (2016), aplicaron el MF a 550 universitarios para reducir el distrés durante el período de exámenes, incrementar la resiliencia, y mejorar el desempeño académico de los estudiantes. Obtuvieron que el MF si produjo los resultados esperados en contextos reales, pero no en circunstancias ideales. Rooks, Morrison, Goolsarran, Rogers, Amishi, \& Jha1 (2017) compararon el entrenamiento en MF ( $\mathrm{n}=56)$ con un entrenamiento en relajación $(n=44)$ en el bienestar emocional en un grupo de atletas de alto rendimiento. No se encontraron diferencias significadas en el bienestar emocional entre el grupo de MF y el grupo de relajación. Farias y Wikholn (2016) indican que la aplicación del MF en contextos de salud mental ha llevado a la proliferación de una literatura impregnada por la falta de autocrítica conceptual y rigor metodológico.

Maldonado, Rubio \& López (2016), realizaron una revisión de la literatura utilizando la base de datos Cochran, y afirman que no existen suficientes evidencias del MF como tratamiento efectivo del estrés y del dolor. Van Dam et al. (2018), indica que el movimiento del MF y la evidencia empírica que lo respalda no han pasado los criterios de la metodología de la ciencia; señalan que la información errónea y la metodología deficiente del MF pueden hacer que los consumidores se vean perjudicados, engañados y decepcionados. Hyland (2016), afirma que como suele suceder con las innovaciones populares, el creciente interés y atractivo de la práctica del MF ha llevado a un reduccionismo y mercantilización de los principios básicos y fundamentos éticos de dicha práctica que amenaza con subvertir y afectar el logro de los objetivos originales de las intervenciones en general y su función educativa en particular.

La investigación realizada es de tipo aplicativa, dado que el objetivo del presente estudio es evaluar la incidencia del MF en la disminución del estrés académico en un grupo de estudiantes universitarios, planteando las hipótesis del siguiente modo: Ho: La aplicación del MF no influye en la disminución del estrés académico, ocasionadas por diversas inquietudes que preocupan, entre ellas las reacciones físicas y psicológicas en estudiantes de administración y psicología de la Universidad Autónoma del Perú. H1: La aplicación del MF influyen en la disminución del estrés académico, ocasionadas por diversas inquietudes que preocupan, entre ellas las reacciones físicas y psicológicas en estudiantes de administración y psicología de la Universidad Autónoma del Perú. De acuerdo a la revisión de la literatura, nos inclinamos a postular que el MF disminuirá el nivel de estrés académico de los estudiantes universitarios. 


\section{Método}

\section{Diseño}

Se utilizó un diseño cuasi-experimental con pre prueba-post prueba y con grupos intactos de comparación estática, uno de ellos ejerció como grupo experimental control, y el otro como grupo control, ya que existe un antes y un después en la investigación. El estudio fue cuasi-experimental ya que la variable tratamiento (mindfulness) se aplicó al grupo experimental y no se administró al grupo control, y es de carácter explicativo, ya que la investigación se ubica en el tercer nivel de conocimiento por que implica un conocimiento de la teoría, de métodos y técnicas de investigación

\section{Participantes}

La muestra fue de 54 estudiantes de la Universidad Autónoma del Perú, ubicada en Lima sur en el distrito de Villa El Salvador, y se obtuvo mediante un muestreo no probabilístico a criterio; de los cuales mediante una selección interna de forma aleatoria se separaron en dos grupos: el experimental, beneficiarios del programa de mindfulness, y el grupo control sin tratamiento, cada uno con 27 alumnos universitarios inscritos en el ciclo de verano del 2018.

\section{Instrumento}

El Inventario SISCO del Estrés Académico (Barraza, 2008), es un instrumento auto-descriptivo, y un auto-informe que proporciona el estudiante sobre sí mismo o sobre distintos aspectos de su funcionamiento en el aula, basándose en su auto-observación. Este inventario se construyó a partir de los componentes sistémico-procesuales del estrés académico, y está integrado por 41 reactivos tipo Likert que permite identificar la frecuencia en que las demandas son percibidas como estímulos estresores, la frecuencia con que se presentan los síntomas al estímulo estresor. la frecuencia de uso de las estrategias de afrontamientos. El inventario cuenta con una confiabilidad por mitades de .83 y una confiabilidad en alfa de Cronbach de .90 (Barraza, 2007).

\section{Procedimiento}

El Inventario SISCO del Estrés Académico se aplicó a los estudiantes previa información y registro del consentimiento informado. La aplicación de los inventarios se realizó en forma grupal y tuvo una duración promedio de 15 minutos, y se realizó en el aula de clases. Posteriormente se procedió a la asignación al azar a las dos condiciones de grupo, grupo experimental y grupo control respectivamente, siendo el primero de los designados el que recibió el programa de mindfulness durante ocho sesiones una vez a la semana (dos meses), mientras el grupo control tuvo el tiempo libre para realizar tareas propias de su escolaridad. Se utilizó los vídeo de Yolando Calvo Gómez "Mindfuness, primeros pasos" (youtube.com/watch? $\mathrm{v}=-\mathrm{TA} 61 \mathrm{JeXBDU} \& \mathrm{t}=15 \mathrm{~s}$ ) y "Mindfulness emocional" (youtube.com/watch?v=6hTEyWyFLEQ\&t=791s), de 13 y 15 minutos respectivamente, durante todas las sesiones semanales en un ambiente alfombrado, con colchonetas y en posición de acostados. Finalmente, durante cinco minutos escuchaban un vídeo de "Mindfulness music" para la lograr mayor concentración y relajación con esta música de meditación con atención plena (youtube.com/watch?v=EkbM5EfFyME\&t=306s).

\section{Resultados}

Los datos obtenidos se describirán primeramente en función a los índices de confiabilidad del instrumento de medición utilizados para los pretests y postests de los grupos control y experimental, y luego en base a las pruebas estadísticas utilizadas. 


\section{Índices de confiabilidad del Inventario SISCO de Estrés Académico}

Los índices de fiabilidad $\alpha_{1} \mathrm{y} \alpha_{2}$ de cada dimensión del grupo control para las medidas pretest y postest de los reactivos Inquietudes que más preocupan de ocho preguntas son de 0.705 y 0.846 , respectivamente, los que indican que los reactivos tienen un alto grado de confiabilidad; lo mismo para las Reacciones físicas de seis reactivos con índices de 0.832 y 0.721 , tienen un alto grado de confiabilidad; de igual forma para las Reacciones Psicológica de cinco reactivos con índices de 0.848 y 0.720 tienen un alto grado de confiabilidad; así como también para las Reacciones comportamentales de cuatro reactivos con índices de 0.848 y 0.711 tienen un alto grado de confiabilidad; finalmente las Estrategias para enfrentar la preocupación de seis reactivos con índices de 0.639 y 0.832 tienen un considerable grado de confiabilidad. Demostrando así, que en todas las dimensiones de cada par de medidas pretest y postest en el grupo control, se validan sus reactivos de cada característica de estudio para los diferentes tipos de análisis de datos (ver Tabla 1).

Tabla 1. Fiabilidad por dimensiones en el grupo control

\begin{tabular}{|c|c|c|c|c|c|c|c|c|c|c|}
\hline \multirow{2}{*}{$\begin{array}{l}\text { Estadísticas } \\
\text { de fiabilidad } \\
\text { MEDIDAS }\end{array}$} & \multicolumn{2}{|c|}{$\begin{array}{l}\text { Inquietudes que } \\
\text { más preocupan }\end{array}$} & \multicolumn{2}{|c|}{$\begin{array}{l}\text { Reacciones } \\
\text { físicas }\end{array}$} & \multicolumn{2}{|c|}{$\begin{array}{l}\text { Reacciones } \\
\text { psicológicas }\end{array}$} & \multicolumn{2}{|c|}{$\begin{array}{l}\text { Reacciones } \\
\text { comportamentales }\end{array}$} & \multicolumn{2}{|c|}{$\begin{array}{l}\text { Estrategias para } \\
\text { enfrentar la } \\
\text { preocupación }\end{array}$} \\
\hline & $\begin{array}{l}\text { ALFA DE } \\
\text { CRONBACH }\end{array}$ & $\begin{array}{l}\mathrm{N}^{\circ} \\
\text { ITEMS }\end{array}$ & $\begin{array}{l}\text { ALFA DE } \\
\text { CRONBACH }\end{array}$ & $\begin{array}{l}\mathrm{N}^{\circ} \\
\text { ITEMS }\end{array}$ & $\begin{array}{l}\text { ALFA DE } \\
\text { CRONBACH }\end{array}$ & $\begin{array}{l}\mathrm{N}^{\circ} \\
\text { ITEMS }\end{array}$ & $\begin{array}{l}\text { ALFA DE } \\
\text { CRONBACH }\end{array}$ & $\mathrm{N}^{\circ}$ ITEMS & $\begin{array}{l}\text { ALFA DE } \\
\text { CRONBACH }\end{array}$ & $\begin{array}{l}\mathrm{N}^{\circ} \\
\text { ITEMS }\end{array}$ \\
\hline Pretest & .705 & 8 & .832 & 6 & .848 & 5 & .801 & 4 & 639 & 6 \\
\hline Postest & .846 & 8 & .721 & 6 & .720 & 5 & .711 & 4 & .832 & 6 \\
\hline
\end{tabular}

Se observa que la fiabilidad de forma general para el mismo grupo control en cada medida pretest y postest con índices $\alpha_{1}=0.870$ y $\alpha_{2}=0.903$ respectivamente; en dichos grupos se puede determinar que los instrumentos empleados en ambas medidas del grupo control tienen un alto grado de confiabilidad, validando su uso para el análisis de los datos (ver Tabla 2).

Tabla 2. Fiabilidad en forma general en el grupo control

\begin{tabular}{lll}
\hline Medidas & Alfa de Cronbach & No. de elementos \\
\hline Pretest &, 870 & 27 \\
Postest &, 903 & 27 \\
\hline
\end{tabular}

Los índices de fiabilidad $\alpha_{1}$ y $\alpha_{2}$ de cada dimensión para el grupo experimental para cada par de medidas pretest y postest respectivamente son de 0.728 ambos, los que indican que las preguntas para las Inquietudes que más preocupan de ocho reactivos tienen un alto grado de confiabilidad; lo mismo para las Reacciones fisicas de seis reactivos con índices de 0.744 y 0.793 tienen un alto grado de confiabilidad; de igual modo para las Reacciones Psicológica de cinco reactivos con índices de 0.665 y 0.821 tienen un alto grado de confiabilidad; así como también para las Reacciones comportamentales de cuatro reactivos con índices de 0.655 y 0.707 tienen un considerable grado de confiabilidad; finalmente las Estrategias para enfrentar la preocupación de seis reactivos con índices de 0.779 y 0.642 tienen un considerable grado de confiabilidad.

Se demuestra así que en todas las dimensiones de cada par de medidas pretest y postest en el grupo experimental, se validan sus reactivos de cada dimensión de estudio para los diferentes tipos de análisis de datos (ver Tabla 3). 
Tabla 3. Fiabilidad por dimensiones en el grupo experimental

\begin{tabular}{|c|c|c|c|c|c|c|c|c|c|c|}
\hline \multirow{2}{*}{$\begin{array}{l}\text { Estadísticas } \\
\text { de fiabilidad } \\
\text { MEDIDAs }\end{array}$} & \multicolumn{2}{|c|}{$\begin{array}{l}\text { Inquietudes que } \\
\text { más preocupan }\end{array}$} & \multicolumn{2}{|c|}{$\begin{array}{l}\text { Reacciones } \\
\text { físicas }\end{array}$} & \multicolumn{2}{|c|}{$\begin{array}{l}\text { Reacciones } \\
\text { psicológicas }\end{array}$} & \multicolumn{2}{|c|}{$\begin{array}{l}\text { Reacciones } \\
\text { comportamentales }\end{array}$} & \multicolumn{2}{|c|}{$\begin{array}{l}\text { Estrategias para } \\
\text { enfrentar la } \\
\text { preocupación }\end{array}$} \\
\hline & $\begin{array}{l}\text { ALFA DE } \\
\text { CRONBACH }\end{array}$ & $\begin{array}{l}\mathrm{N}^{\circ} \\
\text { ITEMS }\end{array}$ & $\begin{array}{l}\text { ALFA DE } \\
\text { CRONBACH }\end{array}$ & $\begin{array}{l}\mathrm{N}^{\circ} \\
\text { ITEMS }\end{array}$ & $\begin{array}{l}\text { ALFA DE } \\
\text { CRONBACH }\end{array}$ & $\begin{array}{l}\mathrm{N}^{\circ} \\
\text { ITEMS }\end{array}$ & $\begin{array}{l}\text { ALFA DE } \\
\text { CRONBACH }\end{array}$ & $\mathrm{N}^{\circ}$ ITEMS & $\begin{array}{l}\text { ALFA DE } \\
\text { CRONBACH }\end{array}$ & $\begin{array}{l}\mathrm{N}^{\circ} \\
\text { ITEMS }\end{array}$ \\
\hline Pretest & .728 & 8 & .744 & 6 & .665 & 5 & .655 & 4 & .779 & 6 \\
\hline Postest & .728 & 8 & .793 & 6 & .821 & 5 & .707 & 4 & .642 & 6 \\
\hline
\end{tabular}

La fiabilidad de forma general para el grupo experimental en cada medida pretest y postest con índices $\alpha_{1}=$ 0.780 y $\alpha_{2}=0.815$ respectivamente; en dichos grupos se puede determinar que los instrumentos empleados en ambas medidas del grupo experimental tienen un alto grado de confiabilidad, validando su uso para el análisis de los datos (ver Tabla 4)

Tabla 4. Fiabilidad en forma general en el grupo experimental

\begin{tabular}{lll}
\hline Medidas & Alfa de Cronbach & No. de elementos \\
\hline Pretest &, 780 & 27 \\
Postest &, 815 & 27 \\
\hline
\end{tabular}

\section{Prueba de normalidad}

La prueba de la normalidad se realizó para asegurar el comportamiento de la variable aleatoria estrés académico tanto en el grupo control como para el grupo experimental; en la presente investigación se verifica de manera general que el estrés académico tiene un comportamiento de distribución normal, ya que las probabilidades Sig asintóticas (bilateral) para todas las medidas pretest, postest y diferencias de cada grupo control y experimental, resultan mayores a $0.05=5 \%$. Este resultado conduce al uso del estadístico de contraste para la prueba de hipótesis de la investigación, es decir el ANOVA de un factor (ver Tabla 5)

\section{Prueba de homogeneidad}

La prueba de homogeneidad de varianzas complementa a la normalidad, como uno de los requisitos para realizar una comparación entre grupos control y experimental. Entonces como $S i g=0.103$ y dicha probabilidad es mayor al $0.05=5 \%$, entonces se puede demostrar que existe homocedasticidad o dicho de otra manera que las variabilidades entre los grupos control y experimental son iguales. Por lo tanto, al verificar que primero exista normalidad y luego exista homocedasticidad, se puede asegurar la comparación entre los grupos control y experimental (ver Tabla 6) 
Tabla 5. Prueba de Kolmogorov-Smirnov

\begin{tabular}{|c|c|c|c|c|c|c|c|}
\hline \multirow{2}{*}{\multicolumn{2}{|c|}{$\begin{array}{l}\text { Prueba de Kolmogorov-Smirnov para una } \\
\text { muestra }^{\text {a }}\end{array}$}} & \multicolumn{3}{|c|}{ Grupo Control } & \multicolumn{3}{|c|}{ Grupo Experimental } \\
\hline & & Pretest & Postest & Diferencia & Pretest & Postest & Diferencia \\
\hline \multirow[t]{2}{*}{ Parámetros normales ${ }^{\mathrm{b}, \mathrm{c}}$} & Media & 80.26 & 73.96 & -6.30 & 87.41 & 81.67 & -5.74 \\
\hline & $\begin{array}{l}\text { Desviación } \\
\text { estándar }\end{array}$ & 14.303 & 14.535 & 22.487 & 11.212 & 11.682 & 16.582 \\
\hline \multirow{3}{*}{$\begin{array}{l}\text { Máximas diferencias } \\
\text { extremas }\end{array}$} & Absoluta & .109 & .092 & .114 & .175 & .162 & .126 \\
\hline & Positivo & .109 & .092 & .098 & .175 & .162 & .093 \\
\hline & Negativo & -.063 & -.086 & -.114 & -.085 & -.118 & -.126 \\
\hline \multicolumn{2}{|l|}{ Estadístico de prueba } & .109 & .092 & .114 & .175 & .162 & .126 \\
\hline \multicolumn{2}{|l|}{ Sig. asintótica (bilateral) } & $200^{\mathrm{d}, \mathrm{e}}$ & $200^{\mathrm{d}, \mathrm{e}}$ & $200^{\mathrm{d}, \mathrm{e}}$ &, $033^{\mathrm{d}}$ &, $068^{\mathrm{d}}$ & $200^{\mathrm{d}, \mathrm{e}}$ \\
\hline
\end{tabular}

Tabla 6. Prueba de homogeneidad de varianzas

\begin{tabular}{llll}
\hline Estadístico de Levene & df1 & df2 & Sig. \\
\hline 2,756 & 1 & 52 &, 103 \\
\hline
\end{tabular}

En la tabla 7, se puede apreciar que la media del grupo control se encuentra contenido en el intervalo de confianza grupo experimental, lo mismo ocurre para la media del grupo experimental, se encuentra contenida en el intervalo de confianza del grupo control. Esto demuestra una vez más que ambos grupos si son comparables y por lo tanto se pueden contrastar entre ellas.

Requisitos para el tipo de contraste: Si cumple con ambos requisitos de comparación de grupos: Tabla 5. Prueba de distribución de la normalidad y Tabla 6. Prueba de homogeneidad de varianzas. Dado el cumplimiento de los dos requisitos previos de comparación de grupos, entonces se puede realizar el contraste de hipótesis con el ANOva (ver Tabla 7).

En la tabla 8, se tiene el análisis de la varianza ANOVA de un factor, para el contraste de la hipótesis de la investigación, se observa que el valor Sig. $=0.918$ es una probabilidad mayor a la probabilidad planteada en la investigación, que es de 0.05. Entonces como resultados de dicho análisis, se acepta la hipótesis nula, que dice $\mathrm{H}_{0}$ : La aplicación del mindfulness no influye en la disminución del estrés académico, ocasionadas por diversas inquietudes que preocupan, entre ellas las reacciones físicas y psicológicas en estudiantes universitarios. 
Tabla 7. Intervalos de confianza para las medias (95\%).

\begin{tabular}{|c|c|c|c|c|}
\hline Medidas descriptivas & & Grupo control & Grupo experimental & Total \\
\hline $\mathrm{N}$ & & 27 & 27 & 54 \\
\hline Media & & $-6,30$ & $-5,74$ & $-6,02$ \\
\hline Desviación estándar & & 22,487 & 16,582 & 19,571 \\
\hline Error estándar & & 4,328 & 3,191 & 2,663 \\
\hline \multirow{2}{*}{$\begin{array}{l}95 \% \text { del intervalo de confianza para la } \\
\text { media }\end{array}$} & Límite inferior & $-15,19$ & $-12,30$ & $-11,36$ \\
\hline & Límite superior & 2,60 &, 82 &,- 68 \\
\hline Mínimo & & -52 & -49 & -52 \\
\hline Máximo & & 39 & 23 & 39 \\
\hline
\end{tabular}

Tabla 8. Análisis de varianza (ANOVA)

\begin{tabular}{llllll}
\hline & Suma de cuadrados & Gl & Media cuadrática & F & Sig. \\
\hline Entre grupos & 4,167 & 1 & 4,167 &, 011 &, 918 \\
Dentro de grupos & 20296,815 & 52 & 390,323 & & \\
\hline Total & 20300,981 & 53 & & & \\
\hline
\end{tabular}

\section{Discusión}

El MF consiste en focalizar la atención en la situación actual y en el estado físico y psicológico, centrar la conciencia en el medio físico o psicosocial, las emociones y la respiración, o, en comer o caminar. En las últimas dos décadas la investigación en MF indica hallazgos benéficos para la salud en diversos contextos y poblaciones. Sin embargo, muchos científicos indican que a pesar de su popularidad y supuestos beneficios para la salud y el bienestar psicológico, aún faltan datos duros sobre la eficacia del MF en la clínica (Barnes, Hattan, Black \& Schuman, 2017; Cho, Ryu, Noh \& Lee, 2016; Goldberg,Tucker, Greene, Simpson, Kearney \& Davidson, 2017; en el trabajo (D'Abundo, Cara \& Kelly, 2016; Goyal, Singh \& Sibinga, 2014; De Vibe, Solhaug, Tyssen, Friborg, Rosenvinge \& Sørlie, 2013), y la escuela (Miller, Borsatto \& Al-Salom, 2018; Dvoř́áková et al., 2017; Galante, et al., 2016).

Una revisión de la literatura (Goldberg, Tucker, Greene, Simpson, Kearney \& Davidson, 2017), encontró que solo el $10 \%$ de las investigaciones basadas en el MF utilizaron un grupo de control, y que por lo tanto no había forma de contrastar los hallazgos; varios estudios de meta-análisis han obtenido resultados modestos sobre la efectividad del MF en la reducción del estrés, ansiedad, depresión, dolor e ira en poblaciones clínicas y normales, así como en el incremento de la atención y productividad laboral y rendimiento escolar. Varios estudios de revisión de la literatura indican que el MF parecen ser aceptables en el mejoramiento de la salud, pero se requieren investigaciones con mayor rigor metodológico para corroborar los hallazgos (Muñoz, Hoppes, Hellman, Brunk, Bragg, \& Cummins, 2016; Hyland, 2016; Kreplin, Farias, \& Brazil, 2018; Shahidi, Akbari, \& Zargar, 2017).

Los beneficios del MF no son del todo consistentes ni concluyentes. Varios estudios de meta-análisis indican mejoras pequeñas en el estado de ánimo positivo, relaciones sociales satisfactorias, el abuso de sustancias, sueño y la calidad de vida (Van Dam et al., 2018; Rooks, Morrison, Goolsarran, Rogers, Amishi, \& Jha1, 2017; Reavley, 2018). Al compararse el MF con otras técnicas de intervención, como la actividad física y relajación, la atención plena no resultó superior comparativamente (McConville, McAleer, \& Hahne, 2017; O’Driscoll et al, 2017; Rooks, Morrison, Goolsarran, Rogers, Amishi, \& Jha1, 2017). Los hallazgos del MF se publican con entusiasmo y se reportan los resultados con singular alegría y descontrol, fenómeno que se la ha bautizado 
irónicamente como "McMinful” (Holland, Dooley Fedock, Ferebee, Bailey, 2017; Farias, \& Wikholm, 2016; Ghaeni, Hashemian, Mojtabaei, Majdara, \& Aghabeiki, 2017).

Una situación similar sucede con los hallazgos del MF aplicado a los escenarios educativos, donde sin reservas, con un entusiasmo desbordado, y no siempre basados en estudios controlados y basados en evidencias empíricas, se proclama a los cuatro vientos y en forma ligera las bondades y maravillas del MF, como si fuera la panacea para los problemas psicosociales que afectan la adaptación y en rendimiento académico del estudiante, desde los niveles preescolar hasta el universitario.

El estudio realizado fue aleatorio, controlado, con grupos equivalentes, las mediciones del pretest-postest se realizó con un instrumento con fiabilidad externa e interna, la aplicación del programa de MF basado en la reducción de estrés fue estable y consistente durante ocho sesiones (dos meses), en condiciones naturales y normales, el grupo control recibió un tratamiento normal sin mindfulness. En estas condiciones tendría que probarse la eficacia del MF y encontrarse diferencias en la reducción del estrés académico.

Sin embargo, no se encontraron diferencias estadísticamente significativas en la Prueba de KolmogorovSmirnov, al compararse los promedios y desviación estándar del grupo que recibió el programa de MF $(X=81.67$, $D S=11.68)$ con el grupo sin tratamiento $(X=73.96, D S=14.53)$, en la diferencia de medias y diferencias de la $D S$ (grupo experimental: $X=-5.74, D S=16.58$ vs grupo control: $X=-6.30, D S=22.48$ ), y en la diferencia $S i g$. Asintótica (bilateral): ,200 (con MF) vs ,200 (sin MF).

$\mathrm{Al}$ analizar cada grupo por separado, a nivel de intragrupo, se observa que el grupo que recibió el programa de MF no obtuvo diferencias estadísticamente significativas en las mediadas de prestes $(X=87.41, D S=11.212)$ y postest $(X=81.67, D S=11-682$ ), aunque si una ligera disminución en el nivel de estrés académico, pero no significativa estadísticamente hablando. Algo similar se encontró en el grupo control sin tratamiento, como era de esperarse (pretest: $X=80.26, D S=14.303$; postest: $X=73.96, D S=14.535$ ), observándose también una breve disminución del estrés, estadísticamente no significativa, no atribuible al MF ya que este grupo por su condición no recibió la variable experimental.

El análisis de varianza obtenido ( $F=0.11)$ es menor que el valor tabulado $(F=0.918)$, a un nivel de significatividad del 0.05 , lo que demuestra que la administración diferencial del programa de MF para reducir el estrés académico no produjo resultados estadísticamente diferenciales, hallazgos que también coinciden con los puntajes obtenidos en la diferencia de los estadísticos de prueba (grupo MF: $d=.126$; grupo $\sin$ MF: $d=.114$ ), y en la Sig asintótica (bilateral), cuya $d=, 200$ es idéntica en ambos grupos, tal como ya se mencionó anteriormente.

El no haber encontrado influencia del programa de ocho semanas de mindfulness para reducir el estrés académico en un grupo de estudiantes universitarios de psicología y administración, es consistente con algunos resultados de investigaciones donde también se ha encontrado que los programas de mindfulness no tienen un impacto significativo sobre el estrés escolar (Goyal, Singh, \& Sibinga, 2014; Tarrasch, Berman, \& Friedmann, 2016; Golberg et al. 2017; Rooks et al. 2017; Lo, Waterland, \& Todd, 2018; Van Dam et al. 2018; Lynch, Gander, Nahar, Kohls, \& Walach, 2018).

Las dos principales limitaciones del estudio es que la variable estrés académico solo fue evaluada con un solo instrumento, y hubiese sido conveniente haberla evaluado también con otro instrumento que mida lo mismo para ver su consistencia externa. La otra limitación es que la muestra de estudio fue elegida de manera disponible, tal como estaba constituida, aunque la asignación a los grupos si se realizó en forma aleatoria. Como sugerencia para futuras investigaciones recomendamos que toda la selección se haga al azar, que se utilicen al menos dos instrumentos para medir el estrés académico, ampliar la muestra de estudio, y también evaluar el estado general de salud, que es sensible a los altos niveles de estrés en los estudiantes universitarios.

A modo de conclusión, y teniendo en cuenta la revisión de la literatura y los hallazgos del estudio, indicamos que existen resultados contradictorios sobre los beneficios del mindfulness en el ámbito universitario, a favor y en contra; y a pesar de que nuestro hallazgo se ubica en la falta de incidencia de la atención plena en la reducción del estrés académico, consideramos que el dato no se deben tomar en forma concluyente, que haría falta seguir investigando para poder tener resultados concluyentes y definitivos. 


\section{Referencias}

Ali, A., Weiss, T.R., Dutton, A., McKee, D., Jones, K. D., Kashikar, S., Silverman, W.K., \& Shapiro, E.D. (2017). Mindfulness-Based Stress Reduction for Adolescents with Functional Somatic Syndromes. Journal of Pediatrics, 183, 184-190. doi: 10.1016/j.jpeds.2016.12.05

Ahmad, A., Sharif, M., Akbar, A. \& Alavi, M. (2014). Mindfulness and Related Factors among Undergraduate Students. Procedia-Social and Behavioral Sciences, 159, 20-24. doi: 10.1016/j.sbspro.2014.12.321

Barnes, N, Hattan, P. Black, D.S., \& Schuman, Z. (2017). An examination of mindfulness-based programs in US medical schools. Mindfulness, 8, 489-494. doi: 10.1007/s12671-016-0623-8

Barraza, A. (2007). Propiedades psicométricas del Inventario SISCO del estrés académico. Revista PsicologiaCientifica.com, 9, 117-129. Recuperado desde http://www.psicologiacientifica.com/sisco-propiedades-psicometricas

Barraza, A. (2008). El estrés académico en alumnos de maestría y sus variables moduladoras: un diseño de diferencia de grupos. Avances en Psicología Latinoamericana, 26, 270-289. Recovered from http://www.scielo.org.co/pd/ap1/v26n2/v26n2a12.pdf

Broderick, P. C., \& Jennings. P. A. (2012). Mindfulness for adolescents. New Directions for Youth Development, 12, 111-126. doi: 10.1002/yd.20042

Caldwell, K., Harrison, M., Adams, M., Quin, R. H., \& Jeffrey Greeson, J. (2010). Developing Mindfulness in College Students Through Movement-Based Courses. Journal of American College Health, 58, 433-442. doi: 10.1080/07448480903540481

Ching, H.H., Koo, M., Tsai, T.H., \& Chen, C.Y. (2015). Effects of a Mindfulness Meditation Course on Learning and Cognitive Performance among University Students in Taiwan. Evidence-Based Complementary and Alternative Medicine, 25, 17-28. doi: 10.1155/2015/254358

Cho, H., Ryu, S., Noh, J., \& Lee, J. (2016). The Effectiveness of Daily Mindful Breathing Practices on Test Anxiety of Students. PLOS ONE, 11. doi.org/10.1371/journal.pone.0164822

D’Abundo, M. L., Cara, L., \& Kelly A. (2016). The Potential of Promoting Mindfulness in a University Physical Activity and Wellness Course. International Journal of Adult Vocational Education and Technology, 7, 15-45. doi: 10.4018/IJAVET.201601010

De Vibe, M., Solhaug, I., Tyssen, R., Friborg, O., Rosenvinge, J.H., \& Sørlie, T. (2013). Mindfulness training for stress management. BMC Medical Education, 13, 107-115. doi: 10.1186/1472-6920-13-107

Di Pierdomenico, E., Kadziolka, M., \& Carlin, J. (2017). Mindfulness Correlates with Stress and Coping in University Students. Canadian Journal of Higher Education, 47, 2017, 121-134. Recovered from https://files.eric.ed.gov/fulltext/EJ1154090.pdf

Doss, K. \& Bloom, L. (2017). Mindfulness in the middle school classroom: Strategies to target social and emotional well-being of gifted students, Gifted Education International, 34, 2, 181-192. https://doi.org/10.1177/0261429417716352.

Dvořáková, K., Kishida, M., Li, J., Elavsky, S., Broderick, P.C., Agrusti, M.R., \& Greenberg, M.T. (2017). Promoting healthy transition to college through mindfulness training with first-year college students. Journal of American College Health, 65, 259-267. Doi.or $\mathrm{g} / 10.1080 / 07448481.2017 .1278605$

Farias, M., \& Wikholm, C. (2016). Has the science of mindfulness lost its mind? BJPsych Bulletin, 40, 329-332. doi10.1192/pb.bp.116.053686

Galante, J., Dufour, G., Benton, A, Howarth, E., Vainre, M., Croudace, T., Wagner, A.P., Stochl, J., \& Jones, P. (2016). Protocol for the Mindful Student Study. BMJ Open, 6, e012300. doi:10.1136/bmjopen-2016-012300

Galante, J., Dufour, G., Vainre, M., Wagner, A.P., Stochl, J., Benton, A., Lathia, N., Howarth, E., \& Jones, P. B. (2018). A mindfulness-based intervention to increase resilience to stress in university students. The Lancet Public Health, 2, 72-81. doi: 10.1016/ S2468-2667(17)30231-1

Ghaeni, A., Hashemian, K., Mojtabaei, M., Majdara, E., \& Aghabeiki, A. (2017). The effect of mindfulness training (MBSR) on the increasing assertiveness among anxious students. Journal of Urmia University Medical Sciences, 28, 119-129. Recovered from http:// umj.umsu.ac.ir/article-1-3741-en.html

Goldberg, S, B., Tucker, R,P, Greene, P.A, Simpson, T.L., Kearney, D.J., \& Davidson, J.R. (2017). Is mindfulness research methodology improving over time? PLOS ONE, 12, e0187298. doi: 10.1371/journal.pone.0187298

Gouda, S, Luong, M.T, Schmidt, S. \& Bauer, J. (2016). Students and Teachers Benefit from Mindfulness-Based Stress Reduction School-Embedded Pilot Study. Frontiers in Psychology, 7, 590-605. doi: 10.3389/fpsyg.2016.00590

Goyal, M., Singh, S., \& Sibinga, E.M. (2014). Meditation programs for psychological stress and well-being. JAMA, 174, 357-368. doi: 10.1001/jamainternmed.2013.13018

Greeson, J. M., Juberg, M. K., Maytan, M., James, K., \& Rogers, H. (2014). A Randomized Controlled Trial of Koru Journal of American College Health, 62, 222-233. doi: 10.1080/07448481.2014.887571

Holland, A., Dooley, G., Fedock, B., Ferebee S., \& Bailey, L. (2017). Meditation, Mindfulness, and Critical Thinking. Journal of Psychology and Cognition, 2, 170-176. Recovered from http://www.alliedacademies.org/articles/meditation-mindfulness-and-critical-thinkingindividual-characteristics-in-online-higher-education.pdf 
Hj, N., Alavi, M., Mehrinezhad, S. \& Ahmadi, A. (2018). Academic Stress and Self-Regulation among University Students in Malaysia: Mediator Role of Mindfulness, Behavioral Sciences, 8(1), 12; https://doi.org/10.3390/bs8010012

Hyland, T. (2016). The Limits of Mindfulness. British Journal of Educational Studies, 64, 97-117, dOI: 10.1080/00071005.2015.1051946

Ingram, C. M., Breen, A.V., \& van Rhijn, T. (2017). Teaching for well-being?. Journal of Further and Higher Education. doi: 10.1080/0309877X.2017.1409343

Kreplin, U., Farias, M., \& Brazil, I.A. (2018). The limited prosocial effects of meditation. Scientific Reports, 8, 24-33. doi: 10.1038/ s41598-018-20299-z

Li, C., Kee, Y.H. \& Lam, L.S. (2018). Effect of Brief Mindfulness Induction on University Athletes Sleep Quality Following Night Training. Frontiers in Psychology, 9, 508. doi: 10.3389/fpsyg.2018.00508

Lo, K., Waterland, J., \& Todd, P. (2018). Group interventions to promote mental health in health professional education. Advance Health Science Educational, 23, 413-447.doi:10.1007/s10459-017-9770-5

Lynch, S., Gander, M.L, Nahar, A., Kohls, N., \& Walach, H. (2018). Mindfulness-Based Coping With University Life. SAGE Open, 8 , 1-7. doi: $10.1177 / 2158244018758379$

Maldonado, M., Rubio, L., \& López, J. (2016). Evidence-based mindfulness. European Psychiatric, 33, 77-95. doi: 10.1016/j.eurpsy.2016.01.2389

Martin, M. (2018). Mindfulness and Transformation in a College Classroom. Adult Learning, 29, 5-10. doi: 10.1177/1045159517744752

McConville, J., McAleer, R., \& Hahne, A. (2017). Effect of Brief Mindfulness Induction on University Athletes' Sleep Quality Following Night Training. Frontiers Psychology, 9, 508-532. doi: 10.3389/fpsyg.2018.00508

Miller, C.J., Borsatto, J., \& Al-Salom, P. (2018). Testing a quick mindfulness intervention in the university classroom. Journal of Further and Higher Education, 6, 87-98. doi: 10.1080/0309877X.2017.1409345

McConville, J., McAleer, R., \& Hahne, A. (2017). Mindfulness training for health profession students-the effect of mindfulness training on psychological well-being, learning and clinical performance of health professional students. Explore, 13, 26-45. foi: 10.1016/j. explore.2016.10.002

Munoz, R.T., Hoppes, S., Hellman, C.M., Brunk, K.L., Bragg, J. E., \& Cummins, C. (2016). The Effects of Mindfulness Meditation on Hope and Stress. Research on Social Work. doi: 10.1177/1049731516674319

O’Driscoll, M., Byrne, S., McGillicuddy, A, Lambert, S., \& Sahm, L.J. (2017). The effects of mindfulness-based interventions for health and social care undergraduate students. Psychology Health Medicine, 22, 851-865. doi: 10.1080/13548506.2017.1280178

Oman, D., Shapiro, S.L., Thoresen, C.E., Plante, T.G., \& Flinders, T. (2010). Meditation Lowers Stress and Supports Forgiveness Among College Students. Journal of American College Health, 56, 569-578. doi: 10.3200/JACH.56.5.569-578

Parcover, J., Coiro, M. J., Finglass, E., \& Barr, E. (2017). Effects of a Brief Mindfulness Based Group Intervention On College Students. Journal of College Student Psychotherapy. doi: 10.1080/87568225.2017.1407722

Pidgeon, A. M., \& Pickett, L. (2017). Examining the differences between university students' levels of resilience on mindfulness, psychological distress and coping strategies. European Scientific Journal (Special Edition), 103-113. Recovered from http://epublications. bond.edu.au/fsd_papers/506

Ramasubramania, S. (2017). Mindfulness, stress coping and everyday resilience among emerging youth in a university setting. International Journal of Adolescence and Youth, 22, 308-231. doi 10.1080/02673843.2016.1175361

Reavley, N. (2018). Mindfulness training in higher education students. The Lancet Public Health, 3, e55-e56. doi: 10.1016/S24682667(17)30241-4

Regehr, C., Glancy, D., Pitts, A. (2013). Interventions to reduce stress in university students. Journal Affect Disorders, 148, 1-11. doi: 10.1016/j.jad.2012.11.026

Rooks, J.D., Morrison, A.B., Goolsarran, M., Rogers, S.L., Amishi, P., \& Jhal, A.P. (2017). The Influence of Mindfulness vs. Relaxation Training on Athletes' Attention and Well-Being over High-Demand Intervals. Journal of Cognitive Enhancement, 1, 141-153. doi: $10.1007 / \mathrm{s} 41465-017-0016-5$

Schonert, K. A., \& Lawlor, M.S. (2010). The Effects of a Mindfulness-Based Education Program on Pre- and Early Adolescents' Well-Being and Social and Emotional Competence. Mindfulness, 1, 137-151. doi: 10.1007/s12671-010-0011-8

Semple, R, Droutma, V, Reid, B. Mindfulness goes to school: things learned (so far) from research and real-world experiences. Psychology in the Schools. 2017 Jan; 54(1): 29-52.

Shahidi, S., Akbari, H., \& Zargar, F. (2017). Effectiveness of mindfulness-based stress reduction on emotion regulation and test anxiety in female high school students. Journal of Education and Health Promotion, 6, 87-102. doi: 10.4103/jehp.jehp_98_16

Tarrasch, R., Berman, Z., \& Friedmann, N. (2016). Mindful Reading. Frontiers Psychology, 7, 578-593. doi: 10.3389/fpsyg.2016.00578 
Van Dam, N.T, van Vugt, M.K., Vago, D.R., Schmalzl, L. Saron, C.D., Olendzki, A... Meyer, D.E. (2018). Academic Stress and Self Regulation among University Students in Malaysia. Behavioral Sciences, 8, 12-25. doi: 10.3390/bs8010012

Van Dam, N.T, van Vugt, M.K., Vago, D.R., Schmalzl, L. Saron, C.D., Olendzki, A...Meyer, D.E. (2018). Mind the Hype. Perspectives in Psychological Sciences, 13, 36-61. doi: 10.1177/1745691617709589

Verweij, H., van Ravesteijn, H., van Hooff, M., Lagro, P., \& Speckens, A. (2018). Does Mindfulness Training Enhance the Professional Development of Residents? Journal General Internal Medical, 33, 429-436. doi: 10.1097/ACM.0000000000002260

Zenner, C., Herrnleben, S., \& Walach, H. (2014). Mindfulness-based interventions in schools. Frontiers in Psychology, 5, 603-621. doi: $10.3389 /$ fpsyg. 2014.00603 